\title{
Redes Neuronales Artificiales aplicadas a la Predicción del Precio del Oro
}

\author{
Fernando Villada, Nicolás Muñoz y Edwin García-Quintero \\ Universidad de Antioquia, Facultad de Ingeniería, Departamento de Ingeniería Eléctrica, Calle 67 No. 53 - \\ 108, Oficina 20-405, Medellín, Colombia (e-mail: fernando.villada@udea.edu.co)
}

Recibido Feb. 3, 2016; Aceptado Mar. 24, 2016; Versión final Abr. 1, 2016, Publicado Oct. 2016

\begin{abstract}
Resumen
En este trabajo se predice el comportamiento del precio del oro mediante un modelo basado en redes neuronales artificiales (RNA). El objetivo del modelo es predecir los precios de cierre diarios del mercado de Londres, los cuales son tomados como referencia por el Banco central de Colombia. Se estudian varias configuraciones de RNA tipo propagación hacia adelante tomando como variables de entrada las series diarias del índice del dólar estadounidense DXY, el índice SP500, los precios del petróleo y los precios del oro. Se entrenan diferentes estructuras de RNA utilizando la serie histórica de datos, donde una parte de los mismos se utiliza para entrenamiento y la restante para la predicción. Los resultados obtenidos muestran un buen desempeño del modelo tanto en el periodo histórico analizado como en la predicción, encontrando como mejor estructura aquella que utiliza las series de precios diarias del oro, el índice DXY y el índice SP500.
\end{abstract}

Palabras clave: mercados financieros; mercado del oro; redes neuronales; predicción de precios

\section{Artificial Neural Networks applied to the Prediction of the Gold Price}

\begin{abstract}
Gold price prediction using an artificial neural network model (ANN) is proposed in this work. The objective of the model is to predict the daily closing prices in the London market, which are taken as reference prices for the Central Bank of Colombia. Different configurations of type feed-forward ANN are considered using the dollar index DXY, the SP500 index, the daily oil price series, and the daily gold price series, as inputs to the ANN model. A set of ANN structures are trained using the historical series of data, where one portion is used for training and the other portion is used for testing (prediction). The results show good performance of the model both in the analyzed historical period and the predictions, where the best structure includes the daily price series of gold, the DXY index and the SP500 index.
\end{abstract}

Keywords: financial markets; gold exchange market; neural networks; price prediction 


\section{INTRODUCCIÓN}

El estudio del comportamiento futuro del precio del oro es de gran importancia para gobiernos, bancos centrales e inversionistas, quienes utilizan el metal precioso como respaldo a sus monedas o como activo de inversión. El oro al igual que la plata han sido la divisa predominante por más de 4500 años, donde las crisis económicas conocidas desde la antigua Grecia han estado relacionadas con la adulteración de las monedas de oro o con la emisión de divisas (papel moneda) sin el respaldo adecuado en este metal precioso. Por esta razón, el oro ha sido utilizado como activo refugio en épocas de turbulencia económica, para preservar el poder adquisitivo en periodos de alta inflación y como reserva de los países; por lo cual su precio está relacionado fundamentalmente con la demanda de inversionistas y bancos centrales (Bernstein, 2002).

Los principales factores que afectan el precio del oro son: las reservas probadas del metal, los índices de los mercados financieros, los precios del petróleo y factores macroeconómicos (Lili y Chengmei, 2013). Aizenman e Inoue (2013) estudiaron los patrones del negocio del oro por parte de los bancos centrales en el periodo 1979-2010 encontrando sincronía en sus ventas; además de la relación entre la intensidad de las reservas en oro y el poder global de cada país. En la literatura científica se encuentran diferentes modelos matemáticos que estudian el comportamiento del precio del oro. Ismail et al. (2009) usan un modelo de regresión lineal múltiple para predecir el precio del oro en el mes siguiente a partir de ocho variables significativas. Aplicando regresión por pasos para eliminar correlación entre ellas, reducen el modelo a cuatro variables independientes: el índice CRB (Commodity Research Bureau), la tasa de cambio del Euro contra el Dólar estadounidense, la tasa de inflación en los Estados Unidos y el suministro de dinero (M1) en los Estados Unidos; logrando buenos resultados en la predicción.

Con el fin de manejar las volatilidades y no linealidades presentes en el precio del oro, Yaziz et al. (2013) proponen un modelo híbrido ARIMA-GARCH para pronosticar su precio; los resultados obtenidos arrojan errores inferiores a los reportados con modelos lineales. La revisión del mercado global del oro y la tendencia histórica de los precios desde enero de 1968 hasta diciembre de 2008 es presentada en Shafiee y Topal (2010). En el mismo trabajo se aplica un modelo de reversión a la media para estimar el precio futuro en los siguientes diez años encontrándose además una alta correlación entre los precios del oro y del petróleo.

Una revisión detallada de las técnicas de pronóstico de bienes básicos más relevantes publicadas en los últimos diez años es realizada por Ghazali et al. (2012). En dicho trabajo se muestran cuatro tipos de modelos: modelos de regresión lineal, máquinas de soporte vectorial (SVM), modelos ARIMA, modelos GARCH y modelos de inteligencia artificial. Se concluye la superioridad de los modelos de inteligencia artificial basados en redes neuronales pero destacándose que la mayoría de aplicaciones se encuentran en el mercado de la electricidad. Sólo 2 de 34 publicaciones revisadas corresponden a aplicaciones al pronóstico del precio del oro. Otros estudios han analizado el comportamiento cíclico de los precios del oro. En este sentido, Gutiérrez et al. (2013) demuestran que el cociente entre el índice Dow Jones Industrial Average y el precio del oro (DJIA/Oro) es fuertemente cíclico. Los autores utilizan análisis de Fourier para encontrar que el periodo de la relación es de 37 años y de forma complementaria plantean un modelo de redes neuronales artificiales para pronosticar el comportamiento futuro del cociente DJIA/Oro para los años 2011 a 2020, verificando los resultados del modelo con información real de los primeros años del periodo del pronóstico.

El oro al igual que los demás bienes básicos presenta un comportamiento no lineal en su precio. Dado este comportamiento, en los últimos años se ha reportado el uso de nuevos algoritmos basados en redes neuronales artificiales (RNA). La principal ventaja de estas redes de encontrar relaciones no lineales entre las variables de entrada y salida, ha fomentado su aplicación en diferentes ramas de la ingeniería y de los mercados financieros; estos últimos caracterizados por su alta volatilidad (García et al., 2008; Martínez, 2014; Villada et al., 2014; Agudelo et al., 2015). Otras referencias han pronosticado el precio del oro, mostrando un mejor rendimiento de las RNA en comparación con series econométricas y modelos lineales (Parisi et al., 2008; Hussein et al., 2011; Mombeini y Yazdani-Chamzini, 2015). En el último de estos trabajos se estudia el comportamiento mensual del precio del metal precioso mediante modelos ARIMA y RNA, encontrando menores errores en la predicción al utilizar el modelo neuronal. Los indicadores de desempeño utilizados fueron el coeficiente de determinación, el error absoluto medio (MAE) y la raíz del error medio cuadrático (RMSE).

En los proyectos realizados en la línea de mercados energéticos del grupo de investigación en manejo eficiente de la energía de la Universidad de Antioquia (GIMEL), se ha conseguido un mejor rendimiento de las RNA aplicadas a los mercados de valores y de bienes básicos. Por esta razón, el objetivo de esta investigación es desarrollar un modelo de RNA que permita determinar el comportamiento del precio del oro 
en el corto plazo, de tal forma que complemente los trabajos publicados en revistas científicas, más dirigidos al largo plazo. Se estudian varias configuraciones de RNA tomando como variables de entrada las series diarias del índice del dólar estadounidense DXY, el índice S\&P500, los precios del petróleo y los precios históricos del oro. Se entrenan diferentes estructuras de redes neuronales utilizando una serie histórica de datos, donde una parte de éstos se utilizan para entrenamiento y la restante para la predicción. Los resultados obtenidos muestran un buen desempeño del modelo tanto en el periodo histórico analizado como en la predicción.

\section{REDES NEURONALES ARTIFICIALES}

Las redes neuronales artificiales (RNA) son sistemas de aprendizaje inspirados en el funcionamiento del cerebro humano. De esta forma simulan e imitan sistemas permitiendo establecer relaciones no lineales entre las variables de entrada y salida. Su principal ventaja que consiste en procesar información en paralelo en tiempo real ha permitido su aplicación en la clasificación y reconocimiento de patrones en sistemas complejos (Martínez, 2014). El principal potencial de las RNA es detectar no-linealidades en series temporales por lo que han sido de gran utilidad en la predicción de datos económicos y financieros.

En un sistema con RNA los nodos se conectan por medio de sinapsis, esta estructura de conexión determina el comportamiento de la red. La estructura más utilizada es el Perceptrón Multicapa. La figura 1 muestra una estructura de este tipo con dos capas ocultas donde $X_{i}$ representan las entradas, Oi las salidas y $\varphi$ la función de activación. Las capas de entrada dependen de la información disponible para ser clasificada mientras que en las capas de salida se tiene un número de nodos igual a la cantidad de variables de respuesta al medio exterior. Las neuronas en una capa se conectan con las de la capa siguiente mediante sinapsis, cuyo valor es diferente para cada una de las conexiones y se determina a través del proceso de entrenamiento.

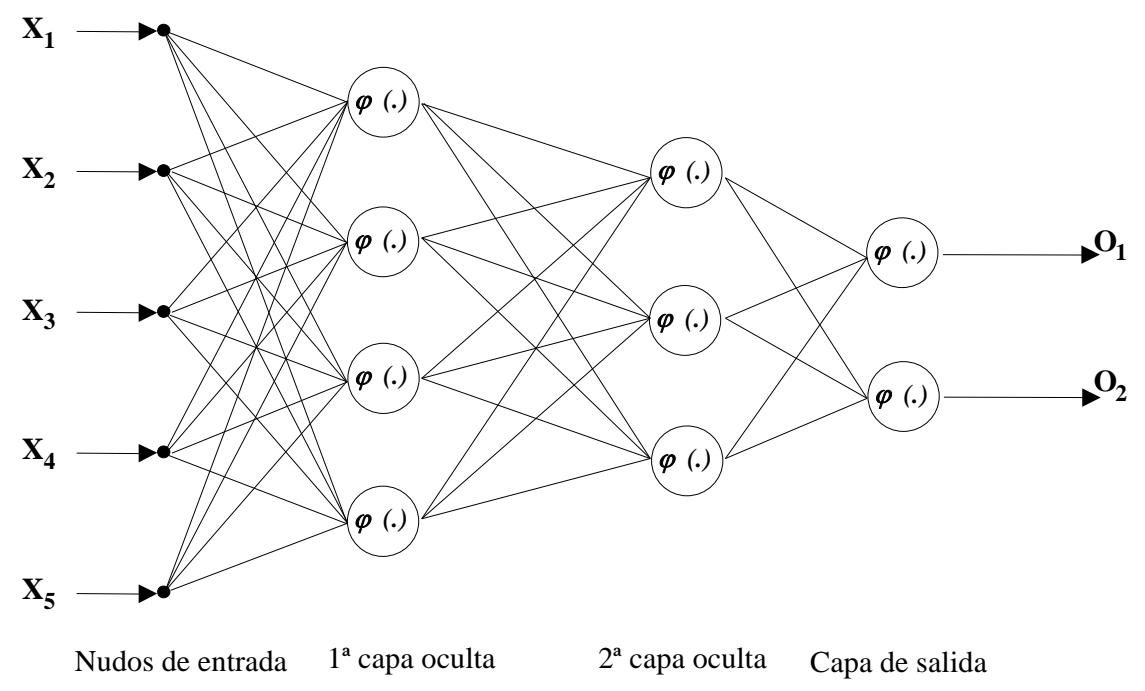

Fig. 1: Estructura de un Perceptrón Multicapa

La expresión matemática que describe la salida de cada neurona se muestra en la ecuación 1 , donde $w_{j i}$ son los pesos sinápticos que ponderan las entradas $x_{i}$ y $\theta_{j}$ es el umbral. $\varphi$ es la función de activación de la neurona y $\mathrm{n}$ es número total de pesos sinápticos conectados a la entrada de la neurona. Cada neurona posee su respectivo nivel de umbral y como función de activación se usó la tangente hiperbólica en todas las neuronas.

$$
y_{i}=\varphi\left(\sum_{i=0}^{n} w_{j i} x_{i}\right)
$$

\section{METODOLOGÍA}

La serie de datos históricos del oro utilizada en este trabajo fue obtenida de los precios diarios fijados en Londres (LBMA, 2015) para el mercado del oro, los cuales se ajustan dos veces al día: AM y PM. También se descargaron de la plataforma de Bloomberg (www.bloomberg.com) los precios del petróleo de referencia para Estados Unidos, la serie de datos del índice del dólar americano (DXY) que pondera diariamente el comportamiento del dólar estadounidense frente a una canasta de las seis monedas más importantes y la 
serie del índice Standard and Poor's 500 (S\&P500) que pondera el comportamiento del precio de las acciones de las 500 empresas más grandes de la bolsa de valores de Nueva York. Las variables de entrada para el primer modelo corresponden a los precios de cierre diarios PM en dólares americanos (referente tomado por el banco central de Colombia), y la salida corresponde al precio a predecir para el día siguiente.

\section{Algoritmo de Aprendizaje}

La regla o algoritmo de aprendizaje es el mecanismo mediante el cual se van adaptando y modificando todos los parámetros de la red. En el caso del perceptrón multicapa se trata de un algoritmo de aprendizaje supervisado; es decir, la modificación de los parámetros se realiza para que la salida de la red sea lo más próxima posible a la salida proporcionada por el supervisor o salida deseada. Por tanto, el proceso de aprendizaje de la red es equivalente a encontrar un mínimo de la función error. El algoritmo de aprendizaje utilizado fue del tipo Levenberg Marquardt del toolbox de redes neuronales de Matlab porque dicho algoritmo en general ha mostrado tener una convergencia más rápida.

\section{Proceso de Aprendizaje}

El objetivo del aprendizaje o entrenamiento de la red, es ajustar los parámetros de la red, pesos y umbrales, con el fin de que las entradas presentadas produzcan las salidas deseadas, es decir con el fin de minimizar la función de error. En lo que respecta al número de capas y neuronas por capa, no existe un método o regla que determine el número óptimo de neuronas ocultas para resolver un problema dado, generalmente se determinan por prueba y error, es decir partiendo de una arquitectura ya entrenada, se realizan cambios aumentando y disminuyendo el número de neuronas ocultas y el número de capas hasta conseguir la arquitectura que se ajuste a la solución del problema.

La RNA utilizada fue del tipo de propagación hacia adelante similar a la mostrada en la figura 1 dada su capacidad como aproximador universal. No existe un procedimiento teórico que permita seleccionar la estructura óptima de RNA. En este caso, se probaron diferentes arquitecturas de una capa oculta, tomando como punto de partida una cantidad de neuronas igual a la semisuma entre el número de entradas y el número de salidas. El proceso de entrenamiento consistió en incrementar paso a paso dicha cantidad de neuronas hasta obtener la arquitectura más adecuada. El criterio de selección de dicha estructura se basó considerando las siguientes medidas de desempeño: MAPE (Error absoluto promedio porcentual, por sus siglas en inglés) y el RMSE (Raíz cuadrada del error promedio cuadrático, por sus siglas en inglés), las cuales se calculan usando las ecuaciones 2 y 3 . Estas dos mediciones de error son las más utilizadas en los textos y artículos científicos dedicados a pronósticos, en especial, Hyndman y Athanasopoulos (2013) destacan la superioridad del RMSE en modelos dependientes de escala y el MAPE cuando hay independencia de escala, recomendando el uso de ambos dada su complementariedad.

$$
\begin{aligned}
& \text { MAPE }=\frac{100}{n} \sum_{t=1}^{n}\left|\frac{y_{t}^{\prime}-y_{t}}{y_{t}}\right| \\
& R M S E=\sqrt{\frac{1}{n} \sum_{t=1}^{n}\left(y_{t}^{\prime}-y_{t}\right)^{2}}
\end{aligned}
$$

En estas ecuaciones, $n$ corresponde a la cantidad de observaciones consideradas, $y_{t}$ es el precio real y $y_{t}^{\prime}$ es el precio obtenido por la red.

El proceso de entrenamiento es supervisado, el cual consiste en presentar un conjunto correspondiente al $62 \%$ de los datos seleccionados de forma aleatoria y modificar iterativamente los pesos hasta obtener un error mínimo deseado en los resultados. Otro conjunto también aleatorio, con el $20 \%$ de los datos es utilizado para validación permitiendo interrumpir el entrenamiento cuando el error de validación empiece a incrementarse y evitar así el sobre entrenamiento. Finalmente, se prueba la estructura de red obtenida con el $18 \%$ de los datos restantes no incluidos en el proceso de entrenamiento.

\section{RESULTADOS Y DISCUSIÓN}

El principal objetivo de este trabajo es atender las necesidades de un operador del mercado del oro de corto plazo, las cuales se refieren a predecir de la mejor forma posible el valor del oro en los siguientes días. En este caso se escogió un horizonte de pronóstico de 22 días hábiles (aproximadamente un mes de negociación), para lo cual se obtuvo una RNA que requería solamente la serie de datos de los cinco meses precedentes. Los resultados del entrenamiento con las medidas de error de las arquitecturas de RNA entrenadas se muestran en la tabla 1 , donde $\mathrm{R}$ designa la cantidad de retardos de tiempo considerados y $\mathrm{Hi}$ 
la cantidad de neuronas en la capa oculta. La serie de datos utilizada para este proceso corresponde a los precios de cierre comprendidos entre el 30 de junio de 2014 y el 30 de diciembre de 2014. Las primeras columnas de la tabla 1 muestran que la RNA puede modelar el precio del oro de forma satisfactoria utilizando su serie de precios como única variable de entrada. El mejor resultado se obtuvo en la configuración 1/5/1 correspondiente a cinco neuronas en la capa oculta y un retardo de tiempo en la capa de entrada, en la cual se tienen bajos errores en el entrenamiento y en la predicción (fuera de la muestra).

Tabla 1: Errores de entrenamiento y predicción del precio del oro con una y dos variables de entrada

\begin{tabular}{|c|c|c|c|c|c|c|c|c|c|}
\hline \multirow{3}{*}{ R } & \multirow{3}{*}{$\mathrm{Hi}$} & \multicolumn{4}{|c|}{$\begin{array}{c}\text { Desempeño de la RNA con una variable de } \\
\text { entrada (serie de precios) }\end{array}$} & \multicolumn{4}{|c|}{$\begin{array}{c}\text { Desempeño de la RNA con dos variables de entrada } \\
\text { (serie de precios e índice } D X Y \text { ) }\end{array}$} \\
\hline & & \multicolumn{2}{|c|}{$\begin{array}{l}\text { Con datos de } \\
\text { entrenamiento }\end{array}$} & \multicolumn{2}{|c|}{ Predicción } & \multicolumn{2}{|c|}{$\begin{array}{l}\text { Con datos de } \\
\text { entrenamiento }\end{array}$} & \multicolumn{2}{|c|}{ Predicción } \\
\hline & & RMSE & MAPE & RMSE & MAPE & RMSE & MAPE & RMSE & MAPE \\
\hline \multirow{4}{*}{1} & 3 & 9.2017 & 0.5286 & 11.2275 & 0.7117 & 8.7829 & 0.5164 & 11.5829 & 0.6687 \\
\hline & 4 & 8.6488 & 0.5139 & 10.9503 & 0.6576 & 8.2115 & 0.4759 & 11.0646 & 0.6488 \\
\hline & 5 & 8.8125 & 0.5112 & 10.7293 & 0.6205 & 8.0579 & 0.4724 & 10.9715 & 0.6174 \\
\hline & 6 & 8.5738 & 0.5072 & 10.9454 & 0.6581 & 7.1196 & 0.4149 & 11.1692 & 0.6231 \\
\hline \multirow{4}{*}{2} & 3 & 8.8402 & 0.5075 & 10.9519 & 0.6819 & 7.9247 & 0.4756 & 10.9457 & 0.6640 \\
\hline & 4 & 8.9163 & 0.5066 & 11.1627 & 0.6854 & 7.3002 & 0.4328 & 10.6751 & 0.6229 \\
\hline & 5 & 8.3419 & 0.4740 & 11.1701 & 0.6769 & 6.6132 & 0.3889 & 10.7003 & 0.6502 \\
\hline & 6 & 8.1190 & 0.4913 & 11.7125 & 0.7338 & 5.9810 & 0.3452 & 10.9545 & 0.6478 \\
\hline \multirow{4}{*}{3} & 3 & 6.5624 & 0.5241 & 11.1478 & 0.7006 & 7.5237 & 0.4417 & 11.5622 & 0.6672 \\
\hline & 4 & 5.8669 & 0.4665 & 11.9477 & 0.7268 & 6.7990 & 0.3964 & 11.3793 & 0.6614 \\
\hline & 5 & 9.4548 & 0.5294 & 12.2456 & 0.7048 & 6.8489 & 0.3902 & 10.8240 & 0.6615 \\
\hline & 6 & 8.4799 & 0.5006 & 11.0798 & 0.7274 & 5.7221 & 0.3219 & 10.7866 & 0.7122 \\
\hline \multirow{4}{*}{4} & 3 & 8.3211 & 0.4844 & 11.5459 & 0.6629 & 8.1724 & 0.4954 & 12.1811 & 0.7478 \\
\hline & 4 & 8.6663 & 0.5126 & 12.4439 & 0.7352 & 6.7791 & 0.3935 & 11.5197 & 0.7244 \\
\hline & 5 & 7.2012 & 0.4194 & 11.7851 & 0.7405 & 5.1251 & 0.2755 & 11.8249 & 0.6914 \\
\hline & 6 & 7.3899 & 0.3966 & 11.1096 & 0.7183 & 6.0595 & 0.3425 & 11.5699 & 0.7158 \\
\hline
\end{tabular}

La mitad derecha de la tabla 1 involucró una segunda variable de entrada que pretende tener en cuenta el hecho de que el oro sea utilizado como principal posición de reserva por parte de la mayoría de los bancos centrales compitiendo con el dólar americano para tal función. La variable escogida fue el índice del dólar estadounidense DXY, el cual se calcula diariamente tomando como referencia la tasa de cambio entre el dólar americano y las seis monedas más transadas en el mundo. Recientemente se ha observado que los grandes inversionistas han buscado refugio en el oro y el dólar de Estados Unidos en los periodos de crisis económica. Tal como se esperaba, la RNA mejoró considerablemente su desempeño al involucrar esta entrada adicional. En las columnas de la derecha de la tabla 1 se aprecia que de forma similar a la primera estructura con una variable de entrada, sobresale en este caso la red 1/5/1 de un rezago de tiempo y cinco neuronas en la capa oculta, arrojando el menor error en la predicción.

Varios autores han resaltado la influencia del comportamiento de los mercados financieros en el precio del oro (Lili y Chengmei, 2013; Shachmurove, 2011); también se han analizado los ciclos de la relación entre el indicador accionario de la bolsa de valores de Nueva York (Dow Jones Industrial Average) y el precio del oro (Gutiérrez et al., 2013) con el fin de predecir su comportamiento futuro. Por esta razón se entrenó otra estructura de red neuronal que incluya al índice Standard and Poor 500 (S\&P500, por sus siglas en inglés) como tercera variable de entrada. Se ha escogido el índice S\&P500 porque es el más representativo del mercado accionario de Nueva York al medir el comportamiento de las 500 empresas más grandes que cotizan en dicho mercado. Las columnas de la mitad izquierda de la tabla 2 muestran el desempeño de la red con esta nueva variable, dando mejores resultados que las anteriores donde se destaca la estructura $1 / 6 / 1$ con el mejor indicador de desempeño en la predicción.

Dado que varias de las referencias consultadas mostraban que el precio del oro era también afectado por el mercado del petróleo, se probó una cuarta estructura de red que incluía tres variables de entrada: los precios del oro, el índice DXY y la serie de precios del petróleo. Los resultados de esta nueva configuración se muestran en las columnas de la mitad derecha de la tabla 2, observándose en forma general menores 
valores en los errores en referencia a los obtenidos en la estructura anterior. Sin embargo, el mejor indicador de desempeño lo siguió presentando la red con las tres entradas correspondientes a la serie de datos de precios del oro y los índices S\&P500 y DXY con seis neuronas en la capa oculta y un solo rezago de tiempo.

Tabla 2: Errores de entrenamiento y predicción del precio del oro con tres variables de entrada

\begin{tabular}{|c|c|c|c|c|c|c|c|c|c|}
\hline \multirow{3}{*}{$\mathrm{R}$} & \multirow{3}{*}{$\mathrm{Hi}$} & \multicolumn{4}{|c|}{$\begin{array}{l}\text { Desempeño de la RNA con tres entradas (serie de } \\
\text { precios del oro, índice S\&P500 e índice DXY) }\end{array}$} & \multicolumn{4}{|c|}{$\begin{array}{c}\text { Desempeño de la RNA con tres entradas (serie } \\
\text { de precios del oro, índice DXY y serie de precios } \\
\text { del petróleo) }\end{array}$} \\
\hline & & \multicolumn{2}{|c|}{$\begin{array}{l}\text { Con datos de } \\
\text { entrenamiento }\end{array}$} & \multicolumn{2}{|c|}{ Predicción } & \multicolumn{2}{|c|}{$\begin{array}{l}\text { Con datos de } \\
\text { entrenamiento }\end{array}$} & \multicolumn{2}{|c|}{ Predicción } \\
\hline & & RMSE & MAPE & RMSE & MAPE & RMSE & MAPE & RMSE & MAPE \\
\hline \multirow{4}{*}{1} & 3 & 7.2770 & 0.4459 & 11.3137 & 0.6470 & 7.3237 & 0.4633 & 11.2929 & 0.6278 \\
\hline & 4 & 8.0822 & 0.4682 & 10.9987 & 0.6217 & 6.8080 & 0.4211 & 10.9790 & 0.6268 \\
\hline & 5 & 6.7818 & 0.4125 & 11.3607 & 0.6318 & 6.8901 & 0.4400 & 10.2412 & 0.6183 \\
\hline & 6 & 7.2853 & 0.4373 & 10.2203 & 0.5908 & 6.4933 & 0.3854 & 10.9554 & 0.6276 \\
\hline \multirow{4}{*}{2} & 3 & 6.6764 & 0.3838 & 11.6639 & 0.6598 & 6.9342 & 0.3951 & 11.1244 & 0.6277 \\
\hline & 4 & 6.9853 & 0.4268 & 11.2934 & 0.6196 & 7.0474 & 0.3893 & 10.9106 & 0.6157 \\
\hline & 5 & 6.1292 & 0.3450 & 10.6722 & 0.6374 & 5.1007 & 0.2939 & 11.2300 & 0.6280 \\
\hline & 6 & 5.1334 & 0.2759 & 10.8954 & 0.6375 & 5.3316 & 0.3075 & 10.4986 & 0.6278 \\
\hline \multirow{4}{*}{3} & 3 & 6.6479 & 0.3800 & 12.0700 & 0.6651 & 6.9895 & 0.4035 & 11.3888 & 0.6612 \\
\hline & 4 & 5.7696 & 0.3317 & 11.1664 & 0.6220 & 6.2704 & 0.3660 & 11.2379 & 0.6406 \\
\hline & 5 & 5.4774 & 0.2920 & 11.8989 & 0.6497 & 5.5084 & 0.2873 & 11.0348 & 0.6210 \\
\hline & 6 & 5.3631 & 0.3053 & 8.2599 & 0.6872 & 5.6455 & 0.3000 & 11.2794 & 0.6583 \\
\hline \multirow{4}{*}{4} & 3 & 6.4859 & 0.3572 & 11.4550 & 0.6276 & 6.9636 & 0.3911 & 11.2514 & 0.6119 \\
\hline & 4 & 5.5695 & 0.2888 & 11.9709 & 0.6923 & 6.5489 & 0.3412 & 12.0541 & 0.6910 \\
\hline & 5 & 4.7033 & 0.2943 & 11.6229 & 0.6931 & 5.9363 & 0.2940 & 11.8537 & 0.6712 \\
\hline & 6 & 5.8145 & 0.2947 & 11.5675 & 0.6532 & 4.0448 & 0.2310 & 11.0939 & 0.6456 \\
\hline
\end{tabular}

Las configuraciones obtenidas de RNA mostraron bajos errores tanto con los datos de entrenamiento como en la predicción, arrojando mejores resultados con cinco y seis neuronas en la capa oculta. Los menores errores se obtuvieron para un retardo de tiempo, por lo tanto el utilizar un número mayor de retardos sólo complica el trabajo de entrenamiento sin mejorar el comportamiento de la red. Adicionalmente, se encontró una similitud en la configuración de la RNA obtenida en este trabajo para una variable de entrada con otra publicada previamente por los autores para el pronóstico del petróleo (Villada et al., 2014) con cinco neuronas en la capa oculta. Esta similitud se puede explicar porque el comportamiento de ambos bienes básicos depende del desempeño de los mercados financieros mundiales, tal como se ha podido apreciar recientemente.

El desempeño de la RNA con una variable de entrada y estructura 1/5/1 se muestra en las figuras 2 y 3 , donde se encuentra una buena aproximación entre el precio real y los resultados de la red. La adición del precio del petróleo como entrada mejoró el comportamiento de la RNA, sin embargo, los menores errores se obtuvieron al incluir el índice DXY y el índice S\&P500, donde los errores de predicción obtenidos son menores a los reportados por otros trabajos que utilizan los mismos índices de error (Ismail et al., 2009; Mombeini y Yazdani-Chamzini, 2015). Las figuras 4 y 5 muestran una mejor predicción de la RNA con estos últimos índices como entradas.

Adicionalmente se verificó la aplicabilidad del modelo propuesto en periodos de alto estrés financiero. Para ello se escogió la crisis hipotecaria de los Estados Unidos que empezó el 15 de septiembre de 2008 con la quiebra del banco de inversión Lehman Brothers. De esta forma se utilizó la mejor estructura encontrada con las tres entradas de series de precios del oro y de los índices DXY y S\&P500, usando la misma cantidad de datos de entrenamiento a partir de la fecha de inicio de esta crisis. Los resultados de la red arrojaron unos errores en el entrenamiento $(\mathrm{RMSE}=9.6625$, MAPE $=0.5666)$ y en el pronóstico $(\mathrm{RMSE}=$ 11.9432, MAPE $=0.7105$ ), los cuales, a pesar de ser más altos que los reportados en la tabla 2, siguen estando dentro del rango de errores mostrados en la tabla 1 para una variable de entrada. Estos resultados pueden considerarse como aceptables dada la alta volatilidad en el periodo escogido. 


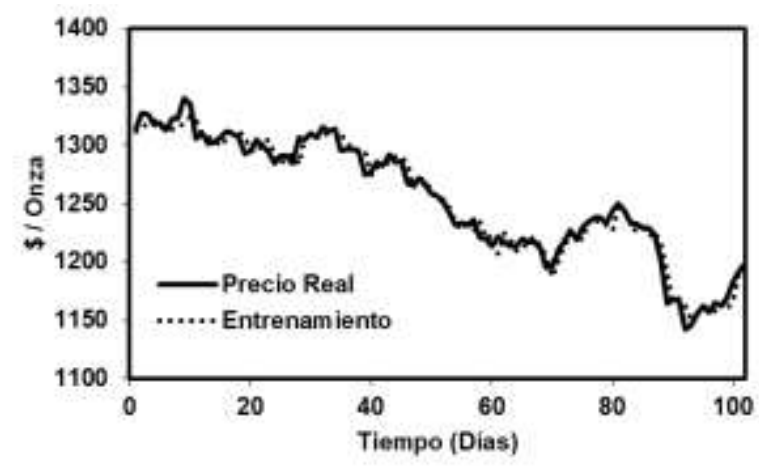

Fig. 2: Desempeño de la RNA con una variable de entrada (datos de entrenamiento)

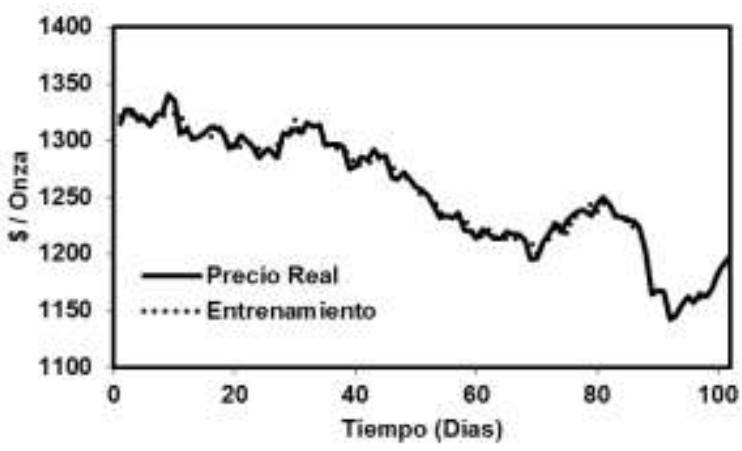

Fig. 4: Desempeño de la RNA con tres entradas (datos de entrenamiento)

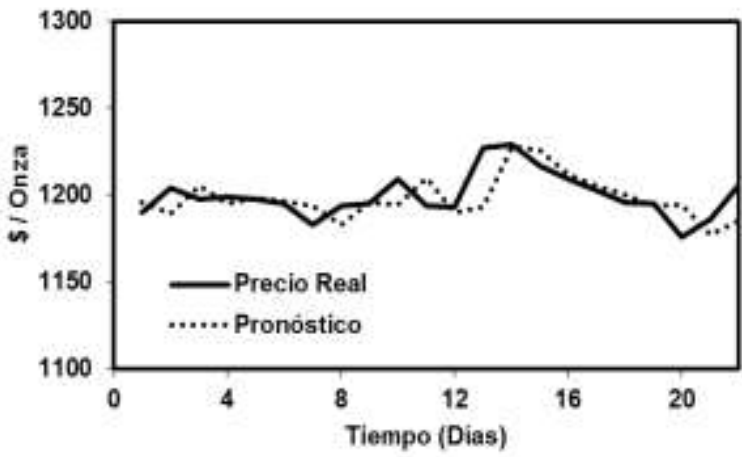

Fig. 3: Desempeño de la RNA con una variable de entrada (predicción)

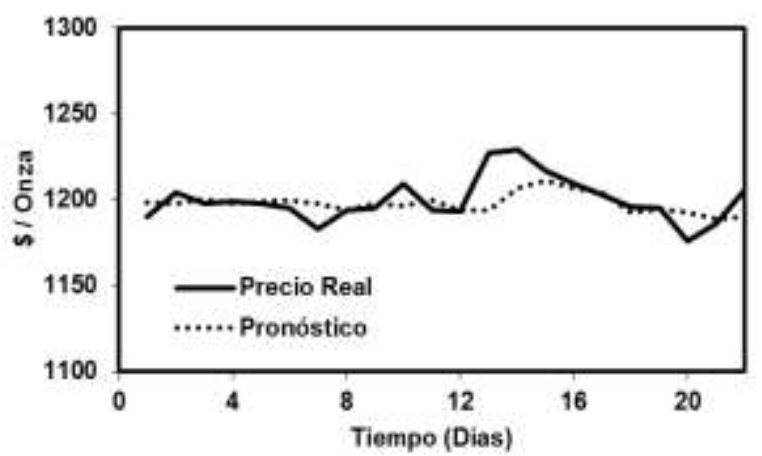

Fig. 5: Desempeño de la RNA con tres entradas (predicción)

\section{CONCLUSIONES}

Los resultados mostrados en la predicción del precio del oro confirman la aplicabilidad de las RNA al mercado de bienes básicos. Se destaca la capacidad de estas redes para modelar sistemas complejos y obtener menores errores tanto en el entrenamiento como en la predicción. El modelo obtenido se convierte en una herramienta de utilidad para gobiernos, bancos centrales y operadores de corto plazo; pues entrega señales de precios que permiten planear compras y ventas del metal precioso con mejor precisión que la arrojada por otros modelos.

Se encontraron mejores resultados al incluir otras variables explicativas que tienen en cuenta la aversión al riesgo. Se verificó el efecto positivo de incluir índices accionarios, sin embargo, se logró mejorar aún más el desempeño al incorporar la serie de precios del oro y los índices DXY y S\&P500 como entradas de la red, logrando menores errores en la predicción en comparación con las referencias consultadas. También se destaca que es suficiente con un rezago en la serie de tiempo para pronosticar satisfactoriamente el precio del oro.

\section{AGRADECIMIENTOS}

Los autores agradecen el apoyo financiero recibido de la Universidad de Antioquia por medio del proyecto "Sostenibilidad 2015-2016".

\section{REFERENCIAS}

Agudelo, A., P., J. M. López-Lezama y E. Velilla, Pronóstico del precio de la electricidad en la bolsa mediante un modelo neuronal no-lineal autorregresivo con entradas exógenas, Revista Información Tecnológica, 26 (6), 99-108 (2015) 
Aizenman, J. y K. Inoue, Central banks and gold puzzles, Journal of the Japanese and International Economics, 28, 63-90 (2013)

Bernstein, P.L., El oro: historia de una obsesión, 1르 edición, 339-357. Javier Vergara Editor, Barcelona, España (2002)

García, I., A. Marbán, Y. M. Tenorio y J. G. Rodríguez, Pronóstico de la concentración de oxono en Guadalajara-México usando redes neuronales artificiales, Revista Información Tecnológica, 19 (3), 89-96 (2008)

Ghazali, M., A. M. Aibinu, J. E. Salami y W. Balogun, A review of forecasting techniques, Proceedings of the 2012 World Congress in Computer Science, Computer Engineering, and Applied Computing, 1-6 (2012)

Gutiérrez, M., G. Franco y C. Campuzano, Gold prices: Analyzing its cyclical behavior, Revista Lecturas de Economía, 79, 113-142 (2013)

Hyndman, R. J. y G. Athanasopoulos, Forecasting: principles and practice. OTexts, Melbourne, Australia (2013)

Hussein, S. F. M., M. B. N. Shah, M. R. A. Jalal y S. S. Abdullah, Gold price prediction using radial basis function neural network, $4^{\text {th }}$ International Conference on Modelling, Simulation and Applied Optimization, 1$11(2011)$

Ismail, Z., A. Yahya y A. Shabri, Forecasting gold prices using multiple linear regression method, American Journal of Applied Sciences, 6(8), 1509-1514 (2009)

LBMA. London Bullion Market Association, http://www.lbma.org.uk/pages/index.html. Consultado el 5 de enero de 2015

Lili, L. y D. Chengmei, Research of the influence of macroeconomic factors on the price of gold, Procedia Computer Science, 17, 737-743 (2013)

Martínez, M., Metodología basada en redes neuronales para interpretación de la resistividad del suelo en zonas urbanas, Ingeniería Energética, 1, 59-69 (2014)

Mombeini, H. y A. Yazdani-Chamzini, Modeling gold price via artificial neural network, Journal of Economics, business and Management, 3(7), 699-703 (2015)

Parisi, A., F. Parisi y D. Díaz, Forecasting gold price changes: Rolling and recursive neural networks models, Journal of International Financial Management, 18, 477-487 (2008)

Shachmurove, Y., A historical overview of financial crises in the United States, Global Finance Journal, 22(3), 217-231 (2011)

Shafiee, S. y E. Topal, An overview of global gold market and gold price forecasting, Resources Policy, 35, 178-189 (2010)

Villada, F., D. Arroyave y M. Villada, Pronóstico del precio del petróleo mediante redes neuronales artificiales, Revista Información Tecnológica, 25 (3), 145-154 (2014)

Yaziz, S. R., N. A. Azizan, R. Zakaria y M. H. Ahmad, The performance of hybrid ARIMA-GARCH modeling in forecasting gold price, Proceedings of the $20^{\text {th }}$ International Congress on Modelling and Simulation, 12011207 (2013) 